\title{
Las videocreaciones como medio de inclusión y mejora del éxito escolar en una educación artístico-musical
}

Enviado: 5 de noviembre de 2021 / Aceptado: 23 de noviembre de 2021 / Publicado: 31 de diciembre de 2021

SABINA CUENCA POVEDA

Universitat Jaume I (Castellón)

al382423@uji.es

0000-0003-4948-6702

DOI 10.24310/IJNE.8.2021.13810

\section{RESUMEN}

En la actualidad la Educación Artística en Educación Primaria, suele plantearse de forma segmentada, subdividiéndose en dos ámbitos o subáreas, el ámbito musical y el plástico-visual, que suelen discurrir por caminos diferentes, y, donde el lenguaje audiovisual queda relegado a tratarse de forma esporádica dentro del ámbito plástico-visual. Este estudio se centra en investigar si la creación de videocreaciones, concebidas como un Proyecto Audiovisual Creativo e Inclusivo y, a su vez, como una obra artística "global", en la que se integran diferentes lenguajes artísticos (musical, plástico y audiovisual) son una práctica que puede mejorar el éxito escolar dentro del Área de Educación Artística en la etapa de Educación Primaria y, con ello, favorecer la inclusión educativa. La metodología empleada en esta investigación es un estudio de caso, centrado en el alumnado de $5^{\circ}$ de E. Primaria del CEIP Villar Palasí de Sagunto, en el que los datos se presentan en diversas tablas y gráficas basadas en la recogida de datos obtenidos a través de las calificaciones del alumnado en dicha área

\section{ABSTRACT}

Videocreations as a means of inclusion and improvement of school succes in a globar artistic-musical education

At present, Artistic Education in Primary Education tends to be considered in a segmented way, being subdivided into two areas or subareas, the musical field and the visual-plastic field, which tend to follow different paths, and, where audiovisual language is relegated to dealing with sporadic form within the visual-visual field. This study focuses on investigating whether the creation of video creations, conceived as a Creative and Inclusive Audiovisual Project and, in turn, as a "global" artistic work, in which different artistic languages (musical, plastic and audiovisual) are integrated are a practice that can improve school success within the Area of Artistic Education in the Primary Education stage and, with it, favor educational inclusion. The methodology used in this research is a case study, focused on the 5th grade students of Primary School of CEIP Villar Pa- 
durante tres cursos escolares, desde el curso 204/15 al 2016/17. Tras la aplicación del Proyecto Audiovisual Creativo Inclusivo, centrado en la creación de videocreaciones y el análisis de los datos recogidos se observa una mejora del éxito académico del alumnado en el área de Educación Artística, incluyendo el alumnado más vulnerable. Así como se manifiesta un descenso en el fracaso escolar en el ámbito de la Educación Musical.

Palabras Clave: Educación inclusiva, éxito escolar, creación artística, música, medios audiovisuales, enseñanza primaria. lasí de Sagunto, in which the data are presented in various tables and graphs based on the collection of data obtained through of the grades of the students in said area during three school years, from the academic year 204/15 to 2016/17. After the implementation of the Inclusive Creative Audiovisual Project, focused on the creation of video creations and the analysis of the data collected, an improvement in the academic success of students in the area of Artistic Education is observed, including the most vulnerable students. As well as a decrease in school failure in the field of Music Education is manifested.

Keywords: Inclusive Education, School success, artistic creation, music, audiovisual media, primary education.

\section{INTRODUCCIÓN: TEMA OBJETO DE ESTUDIO}

"La inclusión educativa responde a la garantía del derecho a una educación de calidad para todos. Busca maximizar la "presencia”, la "participación" y el "éxito académico" de todos" (Crisol, 2019, p.2), es, justo en este último aspecto, el "éxito académico" sobre el que profundiza este artículo, desde una educación artístico-musical basada en la creación de Proyectos Audiovisuales, concretamente videocreaciones, en los que se conjugan los tres lenguajes artísticos (musical, plástico y audiovisual) que se recogen en el currículum de Educación Primaria, buscando así la mejora de la inclusión educativa.

En los últimos años la educación inclusiva está adquiriendo cada vez un papel más relevante, sobre todo desde las propias prácticas educativas que se dan en las escuelas fomentando prácticas de éxito. Éstas deben fundamentarse en el conocimiento adquirido gracias a la investigación (Jorquera, 2017), sobretodo en una investigación en la acción que muestre el progreso de la educación inclusiva desde la propia práctica (Hernández y Ainscow, 2018).

Sin embargo, desde de la Educación Musical estas prácticas de éxito son mucho menores que en el resto de ámbitos (Jorquera, 2017). Así como se consideran insuficientes las investigaciones realizadas en los propios centros para determinar en qué forma se fomenta la inclusión educativa en la Educación Artística (Cabadas, 2018). También, Augustowsky (2019) señala la 
necesidad de seguir investigando de forma empírica cómo puede influir la participación en la creación de proyectos audiovisuales en la inclusión educativa.

Así pues, teniendo en cuenta las carencias señaladas con anterioridad, desde los tres ámbitos artísticos (musical, plástico y audiovisual) se considera necesario ahondar en aspectos relacionados con éstas, realizando una investigación, que arroje luz sobre cómo se puede fomentar la inclusión educativa desde la Educación Artística a través de la creación de proyectos audiovisuales (en concreto videocreaciones) aumentando así la realización de prácticas de éxito desde el ámbito musical. Por tanto, se define el objetivo de este artículo como: Investigar si la realización de videocreaciones en una educación artística global, durante la etapa de educación primaria, puede favorecer la inclusión educativa a través de la mejora del éxito escolar en dicha área.

\section{ANTECEDENTES Y FUNDAMENTACIÓN TEÓRICA}

Para empezar cabe plantearse qué es y en qué consiste la educación inclusiva. No existe una definición única de qué es la educación inclusiva, sino que existen una gran cantidad y variedad de definiciones, de todas ellas se desprende que la educación inclusiva gira entorno a la aceptación, el reconocimiento y el empoderamiento de la diversidad, junto con la defensa de la equidad del alumnado y de la importancia de desarrollar al máximo las capacidades de todos y cada uno de los alumnos, buscando la eliminación de las barreras que impiden la presencia, la participación y el aprendizaje de todo el alumnado (Ainscow, Booth y Dyson, 2006, Ainscow, 2015, Ainscow, 2017 y Echeita, 2019). No debe confundirse el fomento de la educación inclusiva como un proceso de atención educativa enfocada única y exclusivamente hacia el alumnado más vulnerable, aunque esté comprometida con él (Ainscow, Booth y Dyson, 2006 y Ainscow, 2017), sino que la inclusión educativa debe entenderse de forma más amplia y asumir que se trata de dar una respuesta educativa eficaz a todo el alumnado, de cualquier índole y característica (Echeita, 2017).

El camino en la búsqueda de la educación inclusiva ha sido y es, largo. Desde sus inicios en los años noventa hasta la actualidad se han producido muchos cambios y, aunque formalmente se ha avanzado con diversas iniciativas provenientes de Naciones Unidas, así como de otros organismos y organizaciones internacionales, con iniciativas como, por ejemplo, la Agenda 2030 (UNESCO, 2015), es evidente que existe una gran brecha entre las declaraciones de dichos organismos y la realidad en las aulas (Calderón, 2018, Echeita, 2019 y Azorín y Sandoval, 2019).

En este proceso de búsqueda de la inclusión educativa es clave la figura del docente (Marchesi y Hernández, 2019). Unos docentes que, desde sus centros educativos, se implican cada 
vez de forma más activa en dicho proceso (Echeita, 2019 y Arnáiz, 2019) sobre todo, centrándose en mejorar los aspectos organizativos de los centros y en la metodología empleada en el proceso de enseñanza aprendizaje, focalizándose en mejorar la eficacia escolar para todo el alumnado (Arnáiz, 2019).

Así pues, asumiendo la importancia que los docentes juegan en la búsqueda de la inclusión educativa destacaremos la importancia de las prácticas escolares que dichos realizan, sobre todo en las prácticas denominadas de "éxito escolar". En este caso nos centraremos, tanto en el marco teórico como en las prácticas docentes que se realizan dentro de la Educación Artística, asumiendo en ella el desarrollo del lenguaje plástico, musical y audiovisual y la interrelación de los mismos que se pueden producir en la creación de videocreaciones, entendiendo éstas como Proyectos Audiovisuales Creativos Inclusivos.

En primer lugar, destacaremos la importancia que el arte tiene en la educación inclusiva, desde su propio origen entendiendo y asumiendo que el arte es inclusivo en sí mismo (García, 2012) y que, a su vez, fomenta la inclusión no solo educativa sino también la inclusión social (Mundet, Fuentes-Peláez y Pastor, 2017).

En segundo lugar, cabe destacar el papel que la música juega en la educación inclusiva, Olcina (2017) presenta la música como dinamizadora de los procesos de inclusión, pero destacamos a continuación las aportaciones realizadas por Díaz y Moliner (2020):

Como recurso inclusivo, la revisión de la literatura apunta que la música favorece la participación y el aprendizaje de todo el alumnado y especialmente de aquellos tradicionalmente excluidos, como el alumnado perteneciente a grupos culturales minorizados o a los denominados colectivos vulnerables (Díaz y Moliner, 2020, p. 27).

Así como destaca que la educación musical inclusiva puede: "garantizar la presencia, la participación y el aprendizaje de todo el alumnado en contextos educativos ordinarios a través de dos elementos fundamentales: la adaptabilidad y la música como recurso de inclusión.” (Díaz y Moliner, 2020, p 28).

En tercer lugar, señalaremos que cada vez desde el ámbito musical aparecen con más fuerza voces que demandan una educación artístico-musical en la que los docentes de música deben acercarse a los docentes de arte para encontrar un espacio común (Hemsy, 2003) así como las prácticas educativas y su metodología deben ser flexibles, capaces de adaptarse y seguir las tendencias musicales actuales (Cuevas, 2015) donde se realice un trabajo interdisci- 
plinar desde la creatividad (Alsina, Díaz, Giráldez e Ibarretxe, 2009), apostando por prácticas musicales educativas que busquen un diálogo e interacción entre las manifestaciones artísticas de diversos lenguajes artísticos desde un punto de vista creativo (Murillo, 2015a y Murillo 2015b), en las que la educación artística, habitualmente centrada en las artes plásticas y visuales (Acaso, 2013), no se base en la producción y reproducción de diversas técnicas artísticas, considerándose únicamente como la confección de manualidades (Acaso, 2009), sino que se convierta en una actividad metacognitiva que permita entender y construir una concepción propia de la realidad (Acaso, 2013). Así mismo, esta idea de una educación artística global desde una concepción creativa debería tener como objetivo principal la búsqueda de la inclusión educativa (Fernández-Carrión, 2011), que cada vez se ve más impregnada de la utilización de las TIC (también en su vertiente audiovisual) como un recurso para favorecer la inclusión educativa (Bernaschina, 2019).

En ese sentido dentro de las prácticas educativas van surgiendo experiencias en las que se plantea la Educación Artística desde un punto de vista interdisciplinar, en las que se combinan diversos lenguajes artísticos, como por ejemplo la descrita por Botella y Adell (2018) en la que se integran la música, la plástica y la expresión corporal en la etapa de la Educación Secundaria Obligatoria, o incluso experiencias como las que recoge Giner (2017) en su tesis doctoral en la que investiga la creatividad artística de la infancia desde una educación musical interdisciplinar en un Centro Municipal de Arte de Burriana (Castellón) donde implementan un proyecto (Jardín Artístico) para niños en el que se centran en el desarrollo de la creatividad artística desde la música, las artes plásticas y la danza. Así mismo, esta concepción de interdisciplinariedad en y desde la música desde una perspectiva inclusiva la encontramos en experiencias como el proyecto LOVA (La creación de una ópera: vehículo de aprendizaje), donde el objetivo es lograr progresos educativos en diferentes ámbitos a partir de la creación de una ópera, utilizando el trabajo musical como medio integral e inclusivo de educación (Sanahuja, Moliner y Moliner, 2019) así como la investigación realizada por Alsina, Mallol, Alsina (2020) en la que proponen unir el lenguaje plástico con el musical desde el prisma de la educación inclusiva basada en el ABP, en el que se implementa, por ejemplo, la creación musical a partir de un estímulo visual de una obra de arte.

En cuarto lugar, aún con el aumento de este tipo de prácticas interdisciplinares, que asumen la formación integral del alumnado desde la innovación desde el arte (Tigrero, 2020), estas suelen ser más de carácter puntual, y esporádico, no generalizado y, más aún en aquellos casos en los que interviene la creación audiovisual puesto que, según Álvarez-Rodríguez (2019) los proyectos de creación audiovisual en las prácticas educativas formales apenas existen "y, cuan- 
do lo hace, se produce de manera muy marginal o esporádica” (Álvarez-Rodríguez, 2019, p. 213), como por ejemplo las experiencias expuestas por Arcoba (2018) donde se investiga la creación audiovisual desde el videoarte a partir de autorretratos o las investigaciones de Augustowsky (2019), encaminadas al estudio de la creación audiovisual en la infancia. Con respecto al video arte o las videocreaciones aplicadas en el ámbito educativo encontramos estudios como los de Cuéllar y López- Aparicio (2020a y 2020b) en el que presentan el videoarte como una herramienta para la adquisición de las competencias en alfabetización mediática a través de las emociones así como un recurso para el trabajo con el alumnado de altas capacidades.

Por otra parte, dentro de las investigaciones sobre el campo de la creación audiovisual educativa se encuentran otro tipo de investigaciones como las de Bastida, Hernández y Miranda (2020) que se centran en analizar la utilización del vídeo educativo y las características del mismo, centrándose en vídeos no creados por el alumnado.

Por último, resaltaremos la concepción que presentamos en este estudio sobre lenguaje audiovisual, al que podemos considerar como un punto de encuentro entre los diversos lenguajes artísticos (plástico y musical y audiovisual) y a través de ello fomentar la inclusión educativa. A continuación presentamos un mapa conceptual en el que sintetizamos los fundamentos teóricos de este estudio.

Figura 1. Las vide ocreaciones como medio de inclusión desde una educación artísticomusical cr eativa.

Fuente. Elabor ación pr opia

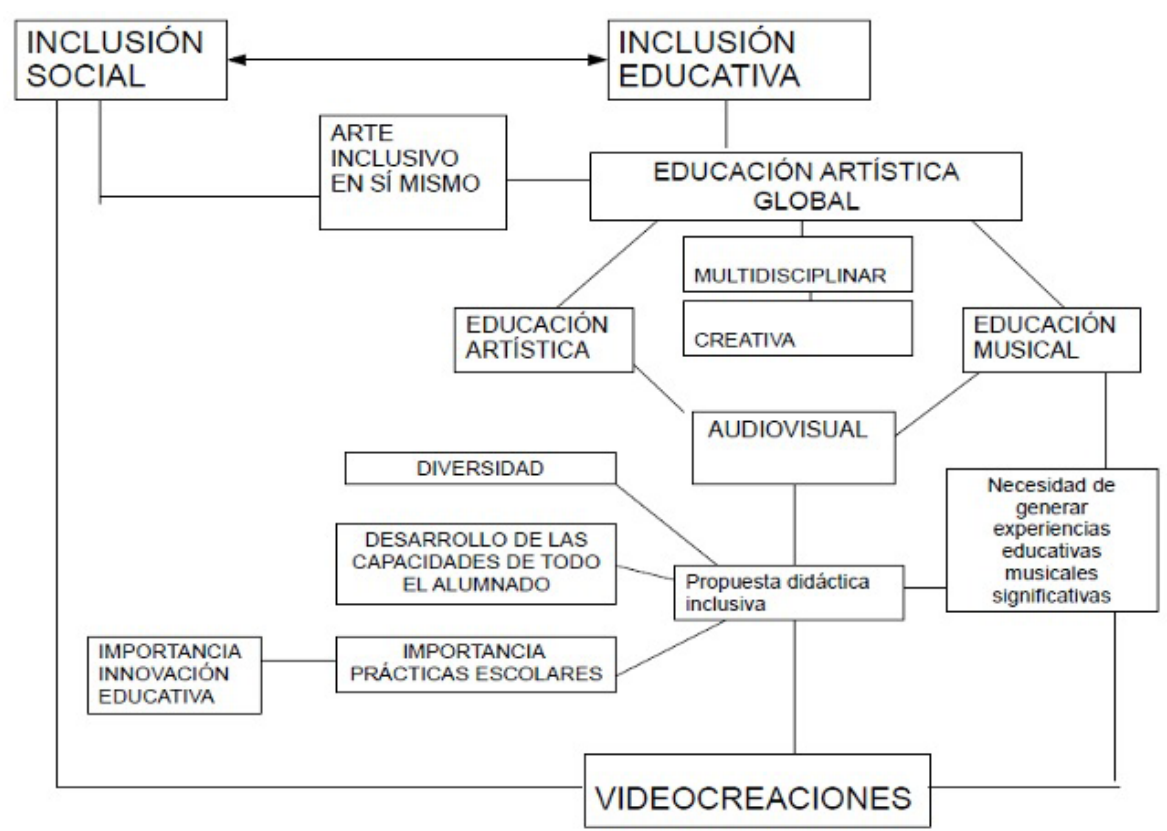




\section{DISEÑO Y METODOLOGÍA}

El presente artículo se encuadraría dentro de la metodología de un estudio de caso, realizado en el CEIP Villar Palasí de Sagunto (Valencia) y que contaría con una propuesta didáctica aplicada en el curso 2015/16 en el área de Educación Artística en el nivel de 5º de Educación Primaria, con un total de 47 alumnos agrupados en 3 grupos-clase, con una carga lectiva de 2 sesiones de 45 minutos semanales, a lo largo del curso escolar 2015/16 (de Octubre de 2015 hasta Junio de 2016).

En este artículo focalizaremos la atención en investigar si la realización de videocreaciones en el área de educación artística mejora el éxito escolar en dicha área. Así pues, los participantes sobre los que se centrará la investigación de este artículo son 39 de los 47 alumnos que realizaron el proyecto, ésto se debe a que los participantes del estudio deben cumplir los siguientes requisitos:

- Alumnado matriculado en el centro el curso anterior a la realización del proyecto, curso 2014/15; en el nivel de $4^{\circ}$.

- Alumnado de nivel de $5^{\circ}$ matriculado en el centro en el curso en el que se desarrolló el proyecto, 2015/16.

- Alumnado matriculado en el centro en el curso posterior a la realización del proyecto, curso 2016/17, en el nivel de $6^{\circ}$.

Se han establecido estos requisitos con el objetivo de poder trazar la evolución del éxito escolar del alumnado para ello, se debe partir del curso anterior a la realización del Proyecto Audiovisual y concluir en el curso posterior a la aplicación del mismo, así pues debe centrarse en el estudio del alumnado en el que se aplicó el proyecto audiovisual en el nivel de $5^{\circ}$ del cual se posee información académica anterior y posterior a la aplicación del proyecto, manteniéndose a lo largo de estos tres cursos en el mismo contexto escolar (incluyendo el mismo grupo-clase).

Para investigar si las videocreaciones pueden mejorar el éxito escolar del alumnado en el área de educación artística se utilizarán, en la recogida de datos, las calificaciones obtenidas por el alumnado en el área de Educación Artística, así como sus subáreas de Educación Musical y Educación Plástica y Visual, entendiendo éstas como un valor objetivo del éxito o fracaso escolar del alumnado, puesto que puede considerarse el rendimiento del alumnado como un indicador indiscutible de inclusión (Karrera, Garmendia y Arguiñano, 2019, p.93). Así pues, se 
han tenido en cuenta en este estudio las calificaciones obtenidas por el alumnado participante en los cursos 2014/15, 2015/16 y 2016/2017.

En este artículo se presentan los resultados de una forma cuantitativa, el procedimiento de análisis de datos se ha basado en la realización de las calificaciones medias obtenidas por el alumnado a lo largo de los diferentes cursos de este estudio, así como también se han realizado porcentajes de algunos aspectos destacables, cómo, por ejemplo, el porcentaje de alumnado que mejora en sus calificaciones, que se mantiene en las mismas o que empeora en los diferentes cursos. Con los datos recogidos se han realizado gráficas y tablas comparativas de las calificaciones medias entre los diversos cursos y de los porcentajes extraídos. Así como, a su vez, encontraremos los datos agrupados en tres grupos. Por una parte los datos del alumnado en general (en los que se incluye todo el alumnado participante en el estudio) y, por otro lado focalizamos la atención en aquellos alumnos más vulnerables, divididos en dos subgrupos, como son el alumnado con problemas de aprendizaje en las áreas instrumentales a los que se le aplican medidas extraordinarias de nivel 4 - ACIS (Adaptación Curricular Individual Significativa) y el alumnado con necesidades de compensación de desigualdades (entre los que se incluyen aquellos alumnos en riesgo de exclusión social, que viven en zonas desfavorecidas, en condiciones sociales y económicas desfavorecidas (tutelados por Servicios Sociales Municipales) y minorías étnicas y culturales). El hecho de analizar los datos agrupados de esta forma responde a la propia concepción de la inclusión educativa en la que se aboga por el aprendizaje de todo el alumnado, prestando especial atención al alumnado más vulnerable (Ainscow, Booth y Dyson, 2006), así como, también a las propias características del centro educativo en el que se realizó este estudio.

El CEIP Villar Palasí de Sagunto escolariza, de forma general, al alumnado que vive en "Racó de l'Horta", una barriada tutelada por el Instituto Valenciano de la Vivienda, donde residen familias con graves necesidades económicas y sociales, hecho que contribuye a rebajar el nivel social y cultural de las familias y, en consecuencia, el del alumnado. Además presenta un alto índice de alumnado de minorías étnicas y culturales, que asciende al $30 \%$ del alumnado, en el momento de la aplicación del proyecto, así como en el nivel que se aplicó el proyecto el $36 \%$ de los participantes en el proyecto presentan dificultades en el aprendizaje de diversa índole, motivo por el que se focalizará en este estudió la evolución del alumnado al que se le aplican medidas extraordinarias de nivel 4 - ACIS.

Puesto que en los resultados se basan en comparaciones de las calificaciones obtenidas entre los diversos cursos académicos, es necesario señalar qué diferencias hay entre unos cursos y otros. 
En el curso 2014/15, anterior a la aplicación del proyecto el Área de Educación Artística se concebía de forma segmentada, la Educación Plástica y la Educación Musical, se trataban como ámbitos artísticos distintos sin puntos en común, que en la práctica podrían, incluso, considerarse como dos áreas completamente independientes.

En el curso 2015/16, curso en el que se aplicó el proyecto el área de Educación Artística se concibe como una área global en la que es la docente de música la que imparte tanto la Educación Plástica como la Educación Musical, concibiendo la misma como una conjunción e interacción entre los tres lenguajes artísticos del currículum de educación primaria (el musical y el plástico-visual, dentro del que se ubica el audiovisual). Para ello, la maestra de música implementó un proyecto audiovisual basado en la creación de videocreaciones, centradas en la ciudad de Sagunto, en el que desde la creatividad del alumnado se creaba tanto la parte visual, la musical y la audiovisual y, todo ello desde el prisma de la inclusión educativa, implementando metodologías pro-inclusivas como el Aprendizaje Basado en Proyectos, el aprendizaje cooperativo, el desarrollo de la creatividad del alumnado, cuidando la direccionalidad y accesibilidad de las actividades diseñadas, para favorecer la presencia, la participación y el aprendizaje de todo el alumnado.

En el curso 2016/17, no se realizó un proyecto de videocreaciones ni ningún otro proyecto audiovisual que incluyera los tres lenguajes artísticos (musical, visual y audiovisual), aunque se siguieron implementando metodologías pro-inclusivas que el alumnado ya había interiorizado.

\section{RESULTADOS}

Gráfica 1. Evolución del índice de alumnado suspendido en el Área de Educación Artística y sus "subáreas" de Educación Musical y Educación Plástica y Visual del curso 2014/15 al 2016/17. Fuente. Elaboración propia basada en las actas de las calificac ones

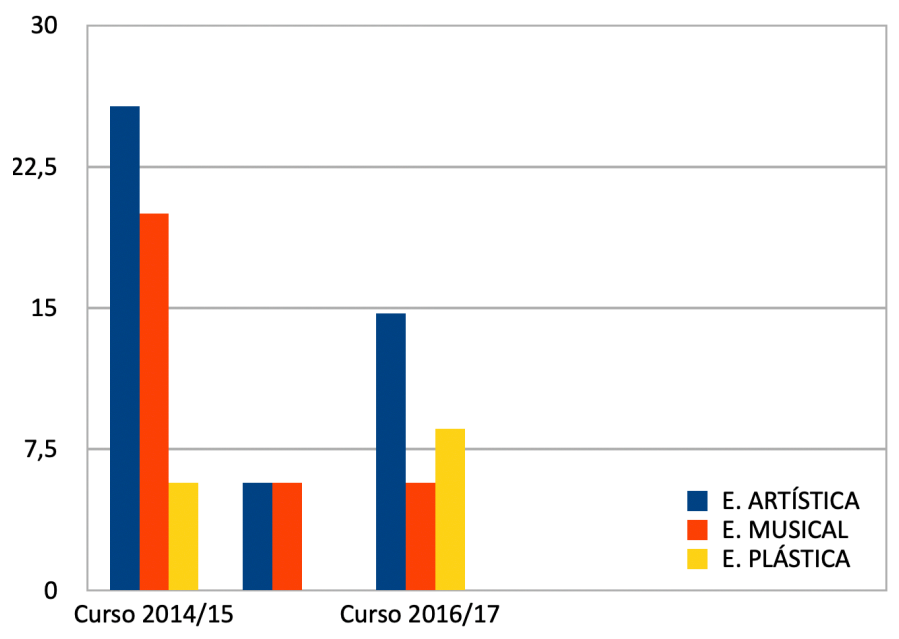


Tabla 1. Evolución del índice de alumnado suspendido en el Ár ea de Educación Artística y sus "sub áreas" de Educación Musical y Educación Plástica y Visual del curso 2014/15 al 2016/17. Fuente. Elaboración pr opia basada en las act as de las calificacione

\begin{tabular}{lccc}
\hline & Curso 2014/15 & Curso 2015/16 & Curso 2016/17 \\
\hline EDUCACIÓN ARTíSTICA & $25^{\prime} 71 \%$ & $5^{\prime} 71 \%$ & $14^{\prime} 71 \%$ \\
\hline EDUCACIÓN MUSICAL & $20,00 \%$ & $5^{\prime} 71 \%$ & $5^{\prime} 71 \%$ \\
\hline EDUCACIÓN PLÀSTICA Y VISU AL & $5^{\prime} 71 \%$ & $0,00 \%$ & $8^{\prime} 57 \%$ \\
\hline
\end{tabular}

Según la gráfica 1 y la tabla 1 se observó como el curso anterior a la aplicación del Proyecto Audiovisual Creativo Inclusivo un 25’71 \% del alumnado suspendía la materia, en el curso 2015/16, curso en el que se aplicó el proyecto, esta cifra descendió hasta el 5’71 \% y en el curso 2016/17 que dejó de aplicarse el proyecto audiovisual inclusivo, se remontó en la cifra de fracaso hasta un $14^{\prime} 71 \%$.

Respecto a la Educación Musical se observó que, en el curso 2014/15 tenía un índice de fracaso del $20 \%$ (del $25 \%$ total del área de E. Artística), mientras que en el siguiente curso, 2015/16 que se aplicó el proyecto, se redujo el fracaso del $20 \%$ al 5,71 \%, manteniéndose el mismo fracaso en el siguiente curso 2016/17, en el que ya no se realizó el proyecto.

En cuanto a la Educación Plástica y Visual se observó como en el curso 2014/15, antes de la aplicación del proyecto, presentaba un 5’71\% de fracaso y que en el curso siguiente 2015/16, en el que se aplicó el proyecto, presentó una mejora del éxito escolar, puesto que ningún alumno/a obtuvo un suspenso en esta subárea. Sin embargo, en el curso 2016/17, en el que ya no se aplicaba el proyecto hubo un repunte en el fracaso y suspendió esta subárea el 8,57 \% del alumnado.

Teniendo en cuenta la gráfica 2 y la tabla 2 (en la siguiente página) sobre la evolución de las calificaciones del alumnado en el área de Educación Artística en el curso 2015/16, curso en el que se implementó el Proyecto Audiovisual Creativo Inclusivo, se observó que la mayoría del alumnado (64’86\%) consiguió mejorar sus calificaciones en el dicha área, que una cifra considerable de alumnado (29'73\%) se mantuvo en sus calificaciones y que un porcentaje muy bajo de alumnado bajó en sus calificaciones (5’4\%).

Respecto al alumnado más vulnerable, como el alumnado con necesidades de compensación de desigualdades (donde se incluyó al alumnado en riesgo de exclusión social, minorías étnicas y culturales, en situación económica desfavorecida, residencia en zonas desfavorecidas con bajos recursos) un alto porcentaje de alumnado (73’68\%) mejoró sus calificaciones, 
lo que representó una mejora considerable respecto a la media, situada en el 63'68\% del alumnado, por lo que se observó que en dicho colectivo se aumentó un $9 \%$ en la mejora de las calificaciones respecto a la media.

Gráfica 2. Evolución de las calificaciones del Á ea de Educación Artística del alumnado del curso 2014/15 al 2016/17 Fuente. Elaboración pr opia

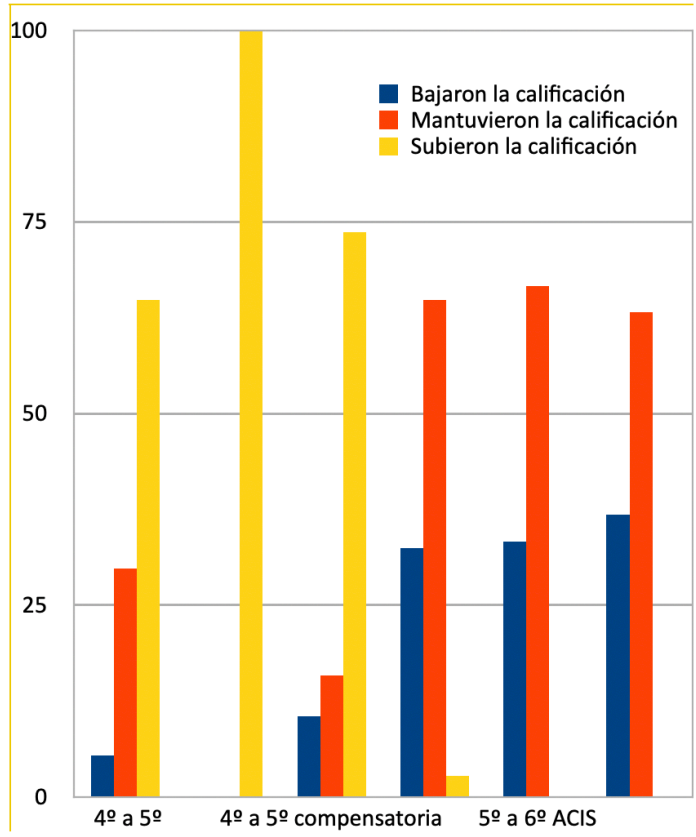

Tabla 2. Evolución de las calificaciones del Á ea de Educación Artística del alumnado del curso 2014/15 al 2016/17. Fuente. Elaboración pr opia

\begin{tabular}{|c|c|c|c|c|}
\hline \multicolumn{2}{|c|}{$\begin{array}{l}\text { DEL CURSO 2014/15 AL 2015/6 (de } 4^{\circ} \text { a } 5^{\circ} \text { ) } \\
\text { Curso en el que se implement ó el pr oyecto 2015/16 (5o) }\end{array}$} & $\begin{array}{l}\text { BAJARON SU } \\
\text { CALIFICACIÓN }\end{array}$ & $\begin{array}{l}\text { MANTUVIERON } \\
\text { SU CALIFICACIÓN }\end{array}$ & $\begin{array}{l}\text { SUBIERON SU } \\
\text { CALIFICACIÓN }\end{array}$ \\
\hline \multicolumn{2}{|l|}{ ALUMNADO } & $5^{\prime} 41 \%$ & $29^{\prime} 73 \%$ & $64 ' 86 \%$ \\
\hline \multirow{2}{*}{$\begin{array}{l}\text { ALUMNADO MÁS } \\
\text { VULNERABLE }\end{array}$} & $\begin{array}{l}\text { ALUMNADO CON ACIS EN ASIGNA - } \\
\text { TURAS INSTRUMENT ALES }\end{array}$ & $0,00 \%$ & $0,00 \%$ & $100,00 \%$ \\
\hline & $\begin{array}{l}\text { ALUMNADO CON COMPENSACIÓN } \\
\text { DE DESIGUALDADES }\end{array}$ & $10^{\prime} 52 \%$ & $15^{\prime} 78 \%$ & $73^{\prime} 68 \%$. \\
\hline
\end{tabular}

\begin{tabular}{|c|c|c|c|c|}
\hline \multicolumn{2}{|c|}{$\begin{array}{l}\text { DEL CURSO 2015/16 AL } 2016 / 7 \text { (de } 5^{\circ} \text { a } 6^{\circ} \text { ) } \\
\text { Curso en el que se implement ó el pr oyecto 2015/16 (5o) }\end{array}$} & $\begin{array}{l}\text { BAJARON SU } \\
\text { CALIFICACIÓN }\end{array}$ & $\begin{array}{l}\text { MANTUVIERON } \\
\text { SU CALIFICACIÓN }\end{array}$ & $\begin{array}{l}\text { SUBIERON SU } \\
\text { CALIFICACIÓN }\end{array}$ \\
\hline \multicolumn{2}{|l|}{ ALUMNADO } & $32^{\prime} 43 \%$ & $64^{\prime} 86 \%$ & $2^{\prime} 71 \%$ \\
\hline \multirow{2}{*}{$\begin{array}{l}\text { ALUMNADO MÁS } \\
\text { VULNERABLE }\end{array}$} & $\begin{array}{l}\text { ALUMNADO CON ACIS EN ASIGNA- } \\
\text { TURAS INSTRUMENT ALES }\end{array}$ & 33 '33 \% & $66^{\prime} 66 \%$ & 0'00 \% \\
\hline & $\begin{array}{l}\text { ALUMNADO CON COMPENSACIÓN } \\
\text { DE DESIGUALDADES }\end{array}$ & $36 ' 84 \%$ & $63^{\prime} 16 \%$ & 0'00\% \\
\hline
\end{tabular}


En cuanto al alumnado al que se le aplicaban medidas de nivel 4, concretamente ACIS (Adaptación Curricular Individual Significativa) en las áreas instrumentales, destacó que el $100 \%$ de dicho alumnado consiguió mejorar su calificación en el área de Educación Artística.

Al comprar los resultados entre el curso en el que se aplicó el Proyecto Audiovisual Creativo Inclusivo, 2015/16, y los resultados obtenidos en el siguiente curso 2016/17 donde no se aplicó el proyecto, se observó que descendió considerablemente la cantidad de alumnado que consiguió mejorar sus calificaciones, al aplicar el proyecto un 64'86 \% consiguió mejorar sus calificaciones, y, por el contrario, al dejar de realizarlo en el siguiente curso únicamente un 2'71 \% del alumnado fue capaz de mejorar su calificación, lo que supuso una diferencia del 62'15 \%. Cabe destacar que, aunque el alumnado en el curso 2015/16 mejoró su calificación seguía quedando margen para mejorar sus calificaciones puesto que éstas no llegaban a la calificación máxima que se podía obtener.

Siguiendo con la comparativa de los datos si fijamos el foco de atención en el alumnado más vulnerable se pudo observar como ningún alumno/a fue capaz de mejorar sus resultados académicos en esta área en el curso en el que no se implementó el Proyecto Audiovisual Creativo Inclusivo, tanto el alumnado con necesidades de compensación de desigualdades como el alumnado con ACIS.

Así mismo, destacó el aumento de alumnado que bajó en sus calificaciones al dejar de implementar el Proyecto Audiovisual Creativo, pasando de un 5’4\% a un 32'43\%, aumentando así en un 27’03 \%. En cuanto al alumnado más vulnerable, el alumnado con necesidades de compensación de desigualdades fue de un 36'84 \%, un 2’5 \% más que la media, y aumentando en un 26’32 \% respecto al curso anterior. En cuanto al alumnado con ACIS fue el que sufrió una mayor bajada en las calificaciones al dejar de implementar el proyecto, puesto que pasó de un $0 \%$ de alumnado que había bajado sus calificaciones cuando se implementó el proyecto a un $33 \%$ de alumnado que bajó sus calificaciones al dejar de realizar el proyecto.

Así mismo, se observó como la mayoría del alumnado (64\%) fue capaz de mantener su calificación una vez se dejó de implementar el proyecto, en cuanto al alumnado más vulnerable las cifras se mantienen cerca de la media (un 66’66 \% de alumnado con ACIS y un $63 \%$ del alumnado con necesidad de compensación de desigualdades). 
Gráfica 3. Evolución de la calificación media del á ea de Educación Artística entr e los cursos 2014/15 y 2016/17. Fuente. Elaboración pr opia basada en las act as de las calificacione

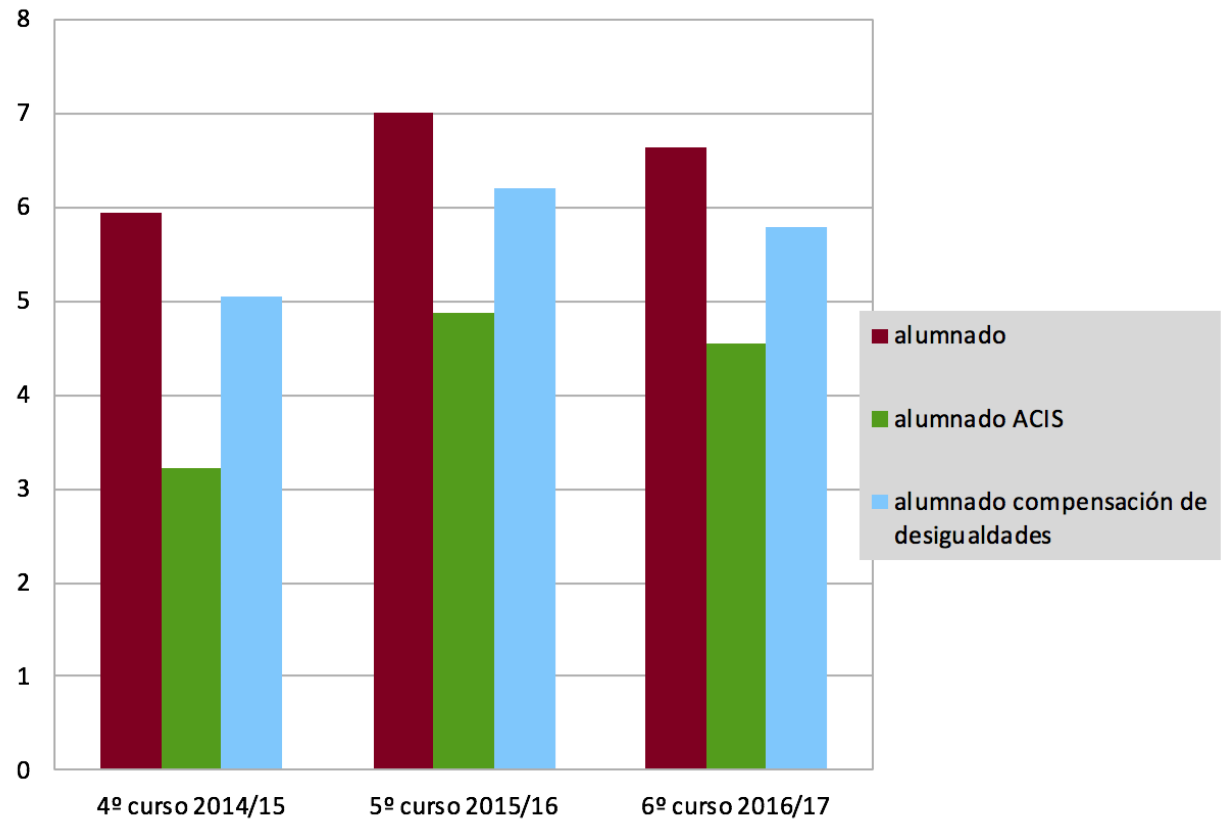

Tabla 3. Evolución de la calificación media delá ea de Educación Artística entr e los cursos 2014/15 y 2016/17. Fuente. Elaboración pr opia basada en las act as de las calificacione

\begin{tabular}{|c|c|c|c|c|}
\hline \multicolumn{2}{|l|}{ CURSOS } & $2014 / 15$ & $2015 / 16$ & 2016/17 \\
\hline \multicolumn{2}{|c|}{ ALUMNADO } & $5 ’ 94$ & 7 & $6{ }^{\prime} 64$ \\
\hline \multirow[t]{2}{*}{$\begin{array}{l}\text { ALUMNADO } \\
\text { MÁS VULNERABLE }\end{array}$} & $\begin{array}{l}\text { ALUMNADO CON ACIS EN ÁREAS } \\
\text { INSTRUMENTALES }\end{array}$ & 3'22 & $4 ' 88$ & $4 ' 55$ \\
\hline & $\begin{array}{l}\text { ALUMNADO CON NECESIDAD DE } \\
\text { COMPENSACIÓN DE DESIGUAL- } \\
\text { DADES }\end{array}$ & $5 ’ 05$ & 6'21 & 578 \\
\hline
\end{tabular}

Según los datos que muestran la gráfica 3 y la tabla 3 se observó cómo del curso 2014/15 al 2015/16 (curso en el que se implementó el proyecto) aumentó la nota media en el área de Educación Artística en un 1'02 puntos. Además, si se pone atención al alumnado más vulnerable se detectó que la nota media del alumnado con ACIS en las áreas instrumentales subió en un 1,66 puntos, superando en 0'64 décimas la nota media general. Así mismo, en el alumnado con necesidades de compensación de desigualdades la nota media aumentó en un 1,16 puntos, mejorando, también, la media general en un 0’14 décimas.

Respecto al siguiente curso 2016/17, en el que no se aplicó el proyecto, se observó una bajada generalizada de la nota media del área de Educación Artística, así como en la nota media 
del alumnado más vulnerable. Aunque no se trató de una baja muy marcada, puesto que no llega a 0’5, sí se pudo observar como la caída de la media general fue del 0’36, en el alumnado más vulnerable con ACIS en las áreas instrumentales fue, prácticamente idéntica, un 0’33 donde se observó un mayor descenso en la nota media fue en el alumnado que presentaba necesidades de compensación educativa, que se situó en un 0’43.

Gráfica 4. Evolución de la calificación media de Educación musical y Educación plástica y visua desde el curso 2014/15 hast a el 2016/17

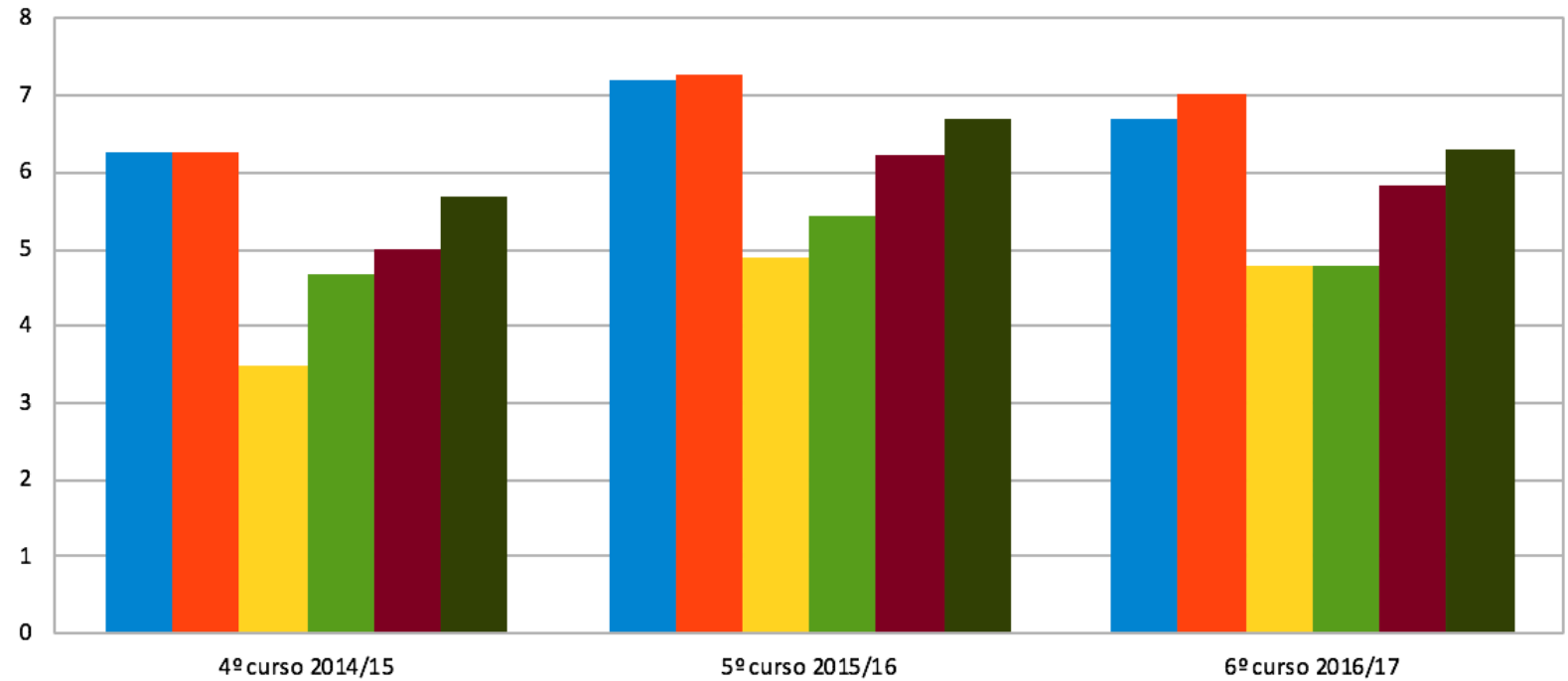

Tabla 4. Evolución de la calificación media de Educación musical y Educación plástica y visual desde el curso 2014/15 has a el 2016/17. Fuente. Elabor ación pr opia b asada en las act as de las calificacione

\begin{tabular}{|c|c|c|c|c|c|c|c|}
\hline \multicolumn{2}{|l|}{ CURSOS } & \multicolumn{2}{|l|}{$2014 / 15$} & \multicolumn{2}{|l|}{$2015 / 16$} & \multicolumn{2}{|l|}{ 2016/17 } \\
\hline & & MÚSICA & PLÁSTICA & MÚSICA & PLÁSTICA & MÚSICA & PLÁSTICA \\
\hline ALUMNADO & & $6^{\prime} 27$ & $6^{\prime} 27$ & $7^{\prime} 21$ & 7'29 & $6 ' 7$ & 7'02 \\
\hline ALUMNADO & ACIS EN ÁREAS & $3^{\prime} 5$ & $4^{\prime} 66$ & $4^{\prime} 88$ & $5^{\prime} 44$ & $4^{\prime} 77$ & $4^{\prime} 47$ \\
\hline \multirow[t]{4}{*}{ MÁS VULNERABLE } & INSTRUMENT ALES & & & & & & \\
\hline & NECESIDAD DE & 5 & $5^{\prime} 68$ & $6^{\prime} 21$ & $6^{\prime} 68$ & $5^{\prime} 84$ & 6'31 \\
\hline & COMPENSACIÓN DE & & & & & & \\
\hline & DESIGUALDADES & & & & & & \\
\hline
\end{tabular}

En los datos que ofrecen la gráfica 4 y la tabla 4 se pudo observar de forma más detallada lo que ocurrió con las 2 subáreas que componen la Educación Artística y que, en el proyecto aplicado se trataron de forma interdisciplinar y relacionada una al servicio de la otra y viceversa. 
En cuanto a lo que se refiere a la subárea de Educación Musical se observó que del curso 2014/15 al 2015/16, en el que se llevó a cabo el proyecto, la nota media general subió un 1’06, mientras que en el alumnado más vulnerable se observó un aumento de la nota media superior a la media general, así pues en el alumnado con ACIS en las asignaturas instrumentales aumentó su nota media en un 1'38 y en el alumnado con necesidades de compensación de desigualdades se situó en una subida de 1'21 de la nota media. Por el contrario, del curso 2015/16 al 2016/17, en el que no se aplicó el proyecto se observó una bajada general en la calificación media de música, que bajó en un 0’51 puntos, por el contrario en el alumnado más vulnerable la bajada de la calificación media fue menor, en el alumnado con necesidades de compensación de desigualdades se situó en una bajada de 0’4, y en cambió en el alumnado con ACIS en las asignaturas instrumentales fue el colectivo en el que la caída fue menor, únicamente un 0’11 puntos.

Respecto a la subárea de Educación Plástica y Visual ocurrió algo similar tanto al área E. Artística general como en la subárea de Educación Musical. Del curso 2014/15 al 2015/16, en el que se aplicó el proyecto, la nota media subió 1'02 puntos, en el alumnado más vulnerable con necesidades de compensación de desigualdades subió 1 punto, y en el alumnado con ACIS en las asignaturas instrumentales subió un 0'85. Del curso 2015/16 al 2016/17 (curso en el que no se implementó el proyecto) se observó una bajada general de las nota media de Educación Plástica y Visual que bajo un 0’27 décimas, mientras que en alumnado más vulnerable se encontró que el alumnado con necesidad de compensación de desigualdades bajo en un 0’37 décimas, y en el alumnado con ACIS en las áreas instrumentales bajo su nota media en 0’67 décimas.

De forma general se observó cómo cuando no se implementó el proyecto la nota media de Educación Musical baja en mayor grado que en la Educación Plástica y Visual, excepto en el colectivo de alumnado vulnerable con ACIS en las áreas instrumentales que baja más en la subárea de Educación Plástica y Visual que en la subárea de Educación Musical, con una diferencia de 0’56 décimas.

\section{DISCUSIÓN DE LOS RESULTADOS}

Los principales hallazgos de este estudio muestran que, entre el alumnado participante, la realización de videocreaciones, concebidas como proyectos audiovisuales creativos globales, en los que se conjugan los tres lenguajes artísticos de la Educación Artística (el lenguaje musical, audiovisual y el plástico-visual), desde una perspectiva basada en la creatividad y la utilización de metodologías pro-inclusivas mejora el rendimiento académico en el área de Educación Artística en la etapa de la educación primaria, así como en los dos ámbitos que la componen 
(Educación Plástica y Visual y Educación Musical). De forma más especifica se observa cómo, el rendimiento académico mejora en mayor grado en el alumnado más vulnerable, como aquel que presenta problemas de aprendizaje (al que se le aplican medidas de nivel 4 - ACIS en las asignaturas instrumentales) y aquel alumnado con necesidad de compensación de desigualdades, entre los que se incluye el alumnado en riesgo de exclusión social, en situación económica y social desfavorecida, en residencia de zonas desfavorecidas, y minorías étnicas y culturales. Además de este estudio se desprende que, cuando la enseñanza de la música se realiza desde una perspectiva global, en la que establece relaciones con otros lenguajes artísticos (como el plástico y el audiovisual) el fracaso en dicho ámbito desciende considerablemente.

Desde La Educación Musical Inclusiva nuestros resultados coinciden con lo apuntado por Díaz y Moliner (2020) que señalan que la revisión de la literatura apunta a que la música favorece el aprendizaje de todo el alumnado, especialmente el del alumnado más vulnerable. En el caso de nuestro estudio ésto se observa desde el ámbito de la práctica escolar, con lo que se respaldaría desde la práctica docente lo que la revisión de la literatura indica.

Así mismo, desde el ámbito de la Educación Musical los resultados obtenidos en nuestro estudio podrían confirmar la hipótesis de Carrera, Román y Chao-Fernández (2017) de que la utilización de las tecnologías (como la grabación musical) en el aula de música puede mejorar el rendimiento académico y la creatividad.

En cuanto al campo del lenguaje audiovisual y la inclusión educativa, nuestros resultados ponen de manifiesto que mediante la utilización del vídeo educativo como recurso inclusivo se puede mejorar el rendimiento escolar, como señalan Bravo-Cobeña, Pin-García, Solís-Pin y Barcia-Zambrano (2021).

Los resultados extraídos de nuestro estudio también pueden poner de manifiesto que la concepción de la Educación Artística entendida como una expresión artística global en la que se fusionan diversos lenguajes artísticos puede potenciar la mejora del éxito escolar en dicha área, como señalan Botella y Adell (2018) que apuntan que esta capacidad de fusión de las artes produce efectos beneficiosos en la adquisición de saberes.

Sin embargo, a diferencia de lo señalado por Muntaner, Pinya y Mut (2020) que indican que el impacto de las metodologías activas en los resultados académicos aún no supone una mejora significativa de los resultados académicos en la educación secundaria, nuestro estudio muestra que, en la Educación Artística la implementación de metodologías activas y proinclusivas sí podrían mostrar una mejora significativa inmediata en la educación primaria. 


\section{LIMITACIONES Y FORTALEZAS DEL ESTUDIO}

Al igual que en la investigación de Muntaner, Pinya y Mut (2020) nuestra investigación también presenta una clara limitación a la hora de establecer el éxito escolar, ésta gira entorno a la utilización de una única variable en la recogida de datos, la de las calificaciones obtenidas por el alumnado, y es que en la actualidad, se trata de la única medida que admite el sistema educativo actual para definir el éxito o el fracaso escolar.

Por el contrario, en este artículo también aparecen diversas fortalezas que destacamos a continuación. En primer lugar, el hecho de que sea la propia maestra de música la que implementa el proyecto audiovisual favorece la inclusión educativa puesto que la música permite conocer mejor al alumnado (Bolívar-Chávez, Véliz-Briones, Alcivar-Cedeño, Zambrano-Sornoza y Cruz-Mendoza, 2018 y Díaz y Moliner, 2020), así como la que lo diseña, puesto que el docente debe ser capaz de diseñar actividades que promuevan la inclusión, donde la música juega un papel fundamental (Bolívar Chávez et al, 2018).

En segundo lugar, destacaremos como fortaleza la aportación que este estudio podría ofrecer al sistema educativo, puesto que se trata de una práctica de éxito a través del audiovisual probada que podría tomarse como referencia para los planes de estudios, idea que defienden Fombona, Pascual y Sevillano (2020), que destacan la necesidad de incorporar al sistema educativo alternativas de éxito tecnológico (incluyendo el audiovisual) probadas para constituir modelos válidos que complementen el sistema educativo actual.

La tercera fortaleza que señalaremos es que afronta uno de los retos que Arnáiz (2019) propone para la mejora educativa y la inclusión, la necesidad de que los propios docentes reflexionen sobre su práctica educativa y que investiguen de forma crítica, puesto que en esta investigación nos encontramos con una docente-investigadora, donde es la propia docente que ha diseñado y aplicado el proyecto audiovisual la investigadora.

\section{CONCLUSIONES}

En conclusión, podemos afirmar que, la realización de videocreaciones, concebidas como Proyectos Audiovisuales Creativos Inclusivos, en el Área de Educación Artística, entendida como la conjunción de los tres lenguajes artísticos que recoge el currículum de la misma en la Educación Primaria (el musical, audiovisual y plástico y visual), mejora el éxito escolar en la propia área, puesto que esta mejora se da de forma general en todos los colectivos, incluyendo los 
más vulnerables. Así pues, la aplicación de dichos proyectos maximiza el éxito escolar en dicha área, así como también, maximiza la presencia y la participación de todo el alumnado gracias a la aplicación de metodologías activas, creativas y pro-inclusivas. Además, de este estudio se desprende que, cuando la enseñanza de la música se presenta dentro de un contexto artístico más amplio, interaccionando con otros lenguajes artísticos el fracaso escolar en dicha subárea desciende considerablemente.

En cuanto a las aplicaciones prácticas de nuestro estudio podemos destacar diversos aspectos. Por una parte, tanto los docentes como las escuelas en general, deberían tener en cuenta los resultados obtenidos en este estudio para replantearse su práctica docente y encaminarla hacia el fomento de la inclusión educativa a través del arte, pudiendo aplicar este tipo de proyectos para mejorar el éxito escolar dentro del área de Educación Artística e incluso extrapolarlo hacia otros ámbitos de conocimiento durante la etapa de Educación Primaria, buscando la transversalidad e interdisciplinariedad. Por otra parte, el propio sistema educativo podría tomar en cuenta los resultados obtenidos para dignificar el área de Educación Artística dentro del mismo, entendiendo el arte y la conjunción de los diversos lenguajes artísticos como un generador y movilizador de aprendizajes potente (Acaso y Megías, 2017) que debería dejar de ocupar un espacio "residual" en nuestro sistema educativo para convertirse en un ámbito con mayor presencia e importancia, sobre todo, si el fin último del sistema educativo es fomentar la educación inclusiva, y poder así favorecer la presencia, la participación y el aprendizaje de todo el alumnado.

\section{REFERENCIAS BIBLIOGRÁFICAS}

Acaso, M. (2009) La Educación artística no son manualidades. Madrid: Los libros de la Catarata.

Acaso, M. (2013) Reduvolution. Barcelona: Paidós.

Acaso, M \& Megías, C. (2017) Art thinking. Barcelona. Paidós.

Ainscow, M.;Booth, T.\& Dyson, A. (2006). Improving schools, developing inclusion. Londres: Routledge.

Ainscow, M. (2015). Struggling for equity in education: the legacy of Salamanca. En F. Kiuppis \& R. S. Hausstätter (Eds.), Inclusive education twenty years after Salamanca (pp. 41-55). New York: Peter Lang.

Ainscow, M. (2017). Pasos para la inclusión en las escuelas. Recuperado de http://www.eduforics.com/ es/pasos-la-inclusion-las-escuelas/

Alsina, P., Díaz, M., Giráldez, A. \& Ibarretxe,G. (2009) 10 Ideas clave El aprendizaje creativo. Barcelona. Graó. 
Alsina, M., Mallol, C. \&Alsina, A. (2020). El reto inclusivo desde la educación artística transformativa. En L. Habib-Mireles (Coord.), Tecnología, diversidad e inclusión: repensando el modelo educativo. (pp. 114-125). Eindhoven, NL: Adaya Press.

Álvarez -Rodríguez, D (2019) La innovación en audiovisuales mediante programas educativos multimodales para la educación primaria. Revista EARI Educación Artística Revista de Investigación, núm 10, 2019, p. 210 -222 recuperado en https://ojs.uv.es/index.php/eari/article/view/13933/14674

Arcoba, M.D (2018) La creación audiovisual en educación artística. Un estudio a partir de autorretratos e identidades desde la videocreación. Tesis Doctoral dirigida por Dr Ricard Huerta Ramón - Universitat de València - Programa de Doctorado de Didácticas Específicas. Departamento de Didáctica de la Expresión Musical, Plástica y corporal. Recuperado en https://roderic.uv.es/ handle/10550/68846

Arnáiz, P. (2019) La educación inclusiva: mejora escolar y retos para el siglo XXI. Revista Participación educativa. Madrid, 2019, segunda época, v. 6, n. 9, septiembre; p. 41-51 Recuperado en https:// redined.educacion.gob.es/xmlui/handle/11162/190717

Augustowsky, G. (2019) La creación audiovisual en la infancia. Estudio de experiencias en contextos educativos. Revista EARI - Educación Artística Revista de Investigación, núm 10 (2019) pp. 235 - 250. Recuperado en https://ojs.uv.es/index.php/eari/article/view/13922/14673

Azorín, C. \& Sandoval, M. (2019). Apoyos para avanzar hacia una educación más inclusiva en los centros escolares: análisis de guías para la acción. Siglo Cero, 50(3), 7. doi: 10.14201/ scero2019503727

Bastida, M., Hernández, F. \& Miranda, J. (2020). “Creación audiovisual educativa: análisis de la producción del Centro de Innovación en Educación Digital (CIED) de la Universidad Rey Juan Carlos”, Paradigmas de la Narrativa Audiovisual. ASRI, 18(16-30). Eumed.net-URJC. Recuperado de https://www.eumed.net/rev/asri//

Bernaschina, D. (2019). Las TIC y Artes mediales: La nueva era digital en la escuela inclusiva. Alteridad, 14(1), 40-52. https://doi.org/10.17163.alt.v14n1.2019.03.

Bolívar-Chávez, O., Véliz-Briones, V., Alcivar-Cedeño, A., Zambrano-Sornoza, J., \& Cruz-Mendoza, J. (2018). La enseñanza de la música. Una estrategia pedagógica para la educación inclusiva. Polo del Conocimiento, 3(12), pp.135-148. doi: $\underline{\text { http://dx.doi.org/10.23857/pc.v3i12.820 }}$

Botella, A. M \& Adell, J. R. (2018) La integración de las artes a través de una propuesta didáctica en educación secundaria obligatoria: música, plástica y expresión corporal. Vivat Academia. Revista de Comunicación. Marzo /junio, 2018, n 142, 109-123. Recuperado en http://www.vivatacademia.net/index.php/vivat/article/view/1062/1312 
Bravo-Cobeña, G.T. , Pin-García, L.A., Solís-Pin, S.C. \& Barcia-Zambrano, A.S. (2021) El video educativo como recursos didáctico inclusivo en la práctica pedagógica actual Polo del Conocimiento, Revista Científico - Académica Multidisciplinaria, Edición núm 54, Vol. 6, nº 1Enero 2021, pp. 201 - 214. Recuperado en https://polodelconocimiento.com/ojs/index.php/es/article/view/2132

Cabadas, V (2018) La Educación Artística como garantía de la inclusión educativa en Educación Primaria. Estudio comparado entre los currículums de Irlanda y España. Actas del XVI Congreso Nacional de Educación comparada de Tenerife, pp 31-30 recuperado en https://riull.ull.es/xmlui/ bitstream/handle/915/11365/1.3.pdf?sequence $=1$

Calderón, I. (2018). Deprived of human rights. Disability and Society, 33(10), 1666-1671. doi: $10.1080 / 09687599.2018 .1529260$

Crisol, E (2019) Hacia una educación inclusiva para todos. Nuevas contribuciones. Profesorado: Revista de currículum y formación del profesorado. Vol 23, nº1 pp. 1- 9 Recuperado en https://recyt. fecyt.es/index.php/profesorado/article/view/72108

Chao-Fernández, A., Pérez Crego, M. C., \& Chao-Fernández, R. (2020). La grabación musical como herramienta de aprendizaje. Implicaciones educativas para el alumnado. Revista de Estudios e Investigación en Psicología y Educación, 7(1), 71-83. https://doi.org/10.17979/reipe.2020.7.1.6520

Cuéllar, F. \& López-Aparicio, I. (2020a). El videoarte como herramienta metodológica y catalizador creativo. La adquisición de las competencias en alfabetización mediática con ayuda de las emociones. [Video art as a methodological tool and creative catalyst. The acquisition of competences in media literacy with the help of emotions]. Vivat Academia. Revista de Comunicación, (151), 127-156. Recuperado de http://www.vivatacademia.net/index.php/vivat/article/ view/1181 [DOI: 10.15178/va.2020.151.127-156]

Cuéllar, F. \&López-Aparicio, I. (2020b). "Investigación del videoarte como recurso narrativo y didáctico en una experiencia con alumnado de altas capacidades” en: Raúl Álvarez \& Mario Rajas (Eds.). Paradigmas de la Narrativa Audiovisuales. ASRI. nº 18: Págs. 70-89. Eumed.net-URJC. Recuperado de https://www.eumed.net/rev/asri/

Cuevas, S (2015) La educación musical y sus avances metodológicos, desde la seunda mitad del siglo XX a la actualidad. Revista Arte y Movimiento. Núm 12, Enero 2015 pp. 40 - 50 Recuperado en https://revistaselectronicas.ujaen.es/index.php/artymov/article/view/1752/3157

Díaz, S \& Moliner, O (2020) Redefiniendo la Educación Musical Inclusiva: Una revisión teórica. Revista Electrónica Complutense de Investigación en Educación Musical, 17, 21-31. Recuperado en http://repositori.uji.es/xmlui/bitstream/handle/10234/190464/71675.pdf?sequence=2 
Echeita, G. (2017). Educación inclusiva. Sonrisas y lágrimas. Aula Abierta, 46, 17-24. doi: 10.17811/ rifie.46.2017.17-24

Echeita, G. (2019) Educación Inclusiva El sueño de una noche de verano. Octaedro Editorial, Barcelona.

Fernández-Carrión, M. (2011). Proyectos musicales inclusivos. Tendencias pedagógicas, (17), 74-82. disponible en https://revistas.uam.es/tendenciaspedagogicas/article/view/1960/2071

Fombona, J., Pascual, $M^{a}$.A. \& Sevillano, $M^{a}$ L. (2020). Construcción del conocimiento en los niños basados en dispositivos móviles y estrategias audiovisuales. Educação \& Sociedade, Revista da Ciencia da Educaçao. Recuperado en https://www.scielo.br/j/es/a/m6hq8hvdgwzNqH4htd5QZ $\underline{\text { bh/?lang=es\&format=pdf }}$

García, C. (2012) “¿Qué puede aportar el arte a la educación? El arte como una estrategia para la inclusión” ASRI- Arte y Sociedad. Revista Investigación №1 Febrero 2012 recuperado en http://www. eumed.net/rev/ays/1/cgm.html

Giner, M. C. (2017) Creatividad artística en la infancia. Una nueva propuesta en Educación Musical. Tesis doctoral de María Carmen Giner Blanco dirigida por Dra. María Fernanda Peset Mancebo. Defendida el 19/09/2017 - Universitat Politècnica de València. Departamento de Comunicación Audiovisual, Documentación e Historia del Arte Recueprada en http://hdl.handle. net/10251/90465

Hemsy, V. (2003). La educación musical entre dos siglos: del modelo metodológico a los nuevos paradigmas. Victoria-Buenos Aires: Universidad de San Andrés. Recuperado en http://live.v1.udesa. edu.ar/files/EscEdu/DT/DT10-GAINZA.PDF

Hernández, A. M. \& Ainscow, M. (2018) Equidad e inclusión: retos y progresos de la escuela del siglo XXI. Revista RETOS XXI, Vol. 2, Núm. 1 (2018) , pp 14 - 22. Recuperado en https://revistas.utp. ac.pa/index.php/retoxxi/article/view/2056/3009

Jorquera, M. ${ }^{a} \mathrm{C}$ (2017) Las buenas prácticas como reflejo del estado de la educación musical: una revisión bibliográfica. Revista Internacional de Educación Musical. №5 pp- 71 - 83. Recuperado en https://dialnet.unirioja.es/servlet/articulo?codigo $=6255058$

Marchesi, A. \& Hernández, L. (2019) Cinco Dimensiones Claves para Avanzar en la Inclusión Educativa en Latinoamérica. Revista Latinoamericana de Educación Inclusiva, 2019, 13(2), 45-56. Recuperado en https://scielo.conicyt.cl/pdf/rlei/v13n2/0718-7378-rlei-13-02-45.pdf

Muntaner, J.J., Pinya, C. \& Mut, B. (2020) El impacto de las metodologías activas en los resultados académicos. Miscelánea, Vol 24 Núm 1, pp. 96-114. DOI: https://doi.org/10.30827/profesorado. v24i1.8846 
Mundet, A., Fuentes-Peláez, N., \& Pastor, C. (2017). El fomento de la inclusión social infantil a través de los lenguajes artísticos. Pedagogía Social. Revista Interuniversitaria,(29),143-156.[fecha de Consulta 24 de Octubre de 2021]. ISSN: 1139-1723. Disponible en: https://www.redalyc.org/ articulo.oa?id=135049901011

Murillo, A (2015a) ¿Más que música? Natura 3.0: diluyendo las fronteras del arte en las aulas de música de secundaria. En Giráldez, A (coord), (2015) De los ordenadores a los dispositivos móviles. Propuestas de creación musical y audiovisual. Barcelona: Graó.

Murillo, A. (2015b) La ciutat com a laboratori: noves cartografies de les pràctiques musicals creatives. Revista Kult-ur, 2 (3), pp. 185-196 Recuperado en http://www.e-revistes.uji.es/index.php/kultur/article/view/1733

Karrera, I, Garmendia, M. \& Arguiñano, A. (2019) Sinergias entre inclusión, competencias y rendimiento escolar: El caso de la escuela pública de Antzuola. Profesorado Revista de currículum y profesorado, Vol. 23 Núm. 1 (2019): Hacia una educación inclusiva para todos. Nuevas contribuciones, Monográfico, Páginas 83-102 DOI: https://doi.org/10.30827/profesorado.v23i1.9145

Sanahuja, A, Moliner, O. \& Moliner, L (2019) Gestión del aula inclusiva a través del proyecto LÓVA: la ópera como vehículo de aprendizaje. Revista Electrónica compluetense de Educación Musical, 16, 3-19 recuperado en http://repositori.uji.es/xmlui/bitstream/handle/10234/184175/66425. pdf? sequence $=1 \&$ isAllowed $=\mathrm{y}$

Tigrero, M. G. (2020). La formación integral de los estudiantes de básica media a través de la innovación desde el arte y la inteligencia emocional. PDR, (18), 35-46. Recuperado a partir de https:// revistas.uniminuto.edu/index.php/Pers/article/view/2286

Unesco. (2015). Educación 2030 Declaración de Incheon y Marco de Acción: hacia una educación inclusiva y equitativa de calidad y un aprendizaje a lo largo de la vida para todos. París: UNESCO. 